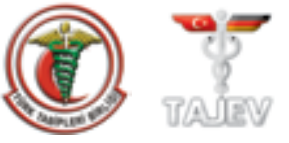

\title{
What do we know about metabolic syndrome in adolescents with PCOS?
}

\author{
Derya Akdağ Cırık, Berna Dilbaz \\ Department of Reproductive Endocrinology and Infertility, Ankara Etlik Zübeyde Hanim Women's Health Education and \\ Research Hospital, Ankara, Turkey
}

\begin{abstract}
Polycystic ovary syndrome (PCOS) is the most common endocrinopathy of reproductive-aged women that manifests itself with a variety of features. For this reason, three different diagnostic criteria have been introduced. For adults, the National Institutes of Health Conference (NIH) criteria, which consists of hyperandrogenism and oligo-anovulation, is the most widely used. Symptoms of PCOS usually start with puberty and may overlap with normal pubertal development. Hormonal fluctuations during this period make the diagnosis of PCOS more difficult. Until now, there is no validated diagnostic criteria for PCOS in adolescents. Although menstrual disorders and cosmetic problems are the most common complaints of adolescents with PCOS, patients should also be evaluated for the potential risk for insulin resistance, obesity, subclinical atherosclerosis, diabetes, metabolic syndrome and cardiovascular disease. Obesity is the most prominent predictor of metabolic syndrome. As the incidence of obesity is increasing both in childhood and adolescence, governments will be faced with a social and economic burden in the future. Adolescents with PCOS are more obese than normal adolescents and have an increased risk of metabolic syndrome. It is suggested that abdominal adiposity increases the risk of metabolic syndrome by inducing various cytokine secretions. Although there is no consensus on metabolic syndrome criteria in the adolescent period, International Diabetes Federation (IDF) criteria may be used for children older than 10 years. Various clinical and metabolic markers are investigated for the prediction of metabolic syndrome in the literature. Waist circumference, serum triglycerides and androgens are the suspected predictors of metabolic syndrome. The prevention of abdominal adiposity and the early diagnosis of PCOS in adolescence should be the main target for the prevention of metabolic syndrome. Clinicians should investigate adolescents with PCOS for metabolic and cardiovascular risks and take preventive action. A Mediterranean diet, low in fat and high in fruits and vegetables, along with moderate-intensity exercise and smoking cessation are the recommended interventions for especially obese adolescents with PCOS. Metformin may be the treatment of choice when lifestyle modifications are ineffective.
\end{abstract}

(J Turk Ger Gynecol Assoc 2014; 15: 49-55)

Key words: Polycystic ovary syndrome, adolescent, metabolic syndrome

Received: 02 December, $2013 \quad$ Accepted: 25 December, 2013

\section{Definition of Polycystic Ovary Syndrome}

Polycystic ovary syndrome (PCOS) was first described by Stein and Leventhal in 1935 (1). The combination of polycystic ovaries and amenorrhoea, with variable hirsutism, were the clinical features of the syndrome. PCOS is a heterogeneous disease that manifests itself with a variety of features; therefore, various definitions are proposed by different working groups. Nowadays, three different diagnostic criteria are currently used for PCOS diagnosis; these are listed in Table 1. According to the Rotterdam Criteria, four phenotypes of PCOS are defined and the groups showed different metabolic characteristics (2). Polycystic ovary was accepted to be an alternative to ovarian dysfunction with the presence of hyperandrogenism, according to the Androgen excess society (AES) criteria; however, a combination of anovulation and polycystic ovaries without hyperandrogenism was not accepted as PCOS (3).
Until now, there is no validated diagnostic criteria for PCOS in adolescents. The Endocrine Society suggested that the diagnosis of PCOS can be made with the presence of persistent oligomenorrhoea and hyperandrogenism (clinical or biochemical) after excluding other pathologies in adolescents (4). Although the National Institutes of Health $(\mathrm{NIH})$ criteria for diagnosis of PCOS is frequently preferred, unexplained hyperandrogenism accompanied by the presence of ovulatory dysfunction is the diagnostic criteria for adolescent PCOS in most cases. However, there is some debate on merely using the Rotterdam-AES criteria (5). The presence of polycystic ovaries in normally ovulating women is a common finding. PCOS can be over-diagnosed in adolescents as the AES criteria accepts the polycystic appearance of the ovaries as evidence of ovulatory dysfunction.

The diagnostic challenge in adolescents may result from many reasons like the higher rate of physiologic anovulatory cycles, irregular menses during the first 2 years following menarche, and the presence of acne at this age group and 
Table 1. Definitions of PCOS

\begin{tabular}{|c|c|c|}
\hline Working Group & Date & Definition \\
\hline NIH (56) & 1990 & Chronic anovulation \& clinical or biochemical hyperandrogenism \& exclusion of other diseases \\
\hline $\begin{array}{l}\text { ESHRE-ASRM / } \\
\text { Rotterdam (57) }\end{array}$ & 2003 & $\begin{array}{l}\text { Presence of at least two of the three criteria: Clinical or biochemical hyperandrogenism, } \\
\text { Oligo-anovulation, Polycystic ovaries }\end{array}$ \\
\hline AES (3) & 2009 & $\begin{array}{l}\text { Hyperandrogenism (hyperandrogenaemia and/or hirsutism) \& ovarian dysfunction } \\
\text { (oligo-anovulation \& polycystic ovaries) \& exclusion of other diseases }\end{array}$ \\
\hline
\end{tabular}

even in "normal" adolescents. Hyperandrogenaemia is thought to be a more reliable diagnostic criteria for the diagnosis of adolescent PCOS after ruling out other causes of hyperandrogenaemia such as late-onset adrenal hyperplasia and Cushing's Syndrome. Moreover, the clinical heterogeneity of the patients who often present with hirsutism without anovulation or anovulation without hirsutism, and the disagreement over whether the polycystic ovarian morphology without hyperandrogenism represents a subgroup of PCOS, leading to a lack of consensus on the diagnostic criteria, especially for adolescents.

\section{Prevalence of PCOS in the Adolescent Population}

Polycystic ovary syndrome (PCOS) is the most common endocrinopathy of reproductive-aged women. While the prevalence of PCOS is given as approximately $7 \%$ by the $\mathrm{NIH}(6)$, it is not easy to estimate the prevalence in adolescents due to the diversity among the experts in this field about the diagnostic criteria and the fact that many symptoms and signs of PCOS may overlap with normal puberty $(7,8)$. Under-diagnosis of PCOS may lead to more serious clinical presentations; for example, women with a diagnosis of PCOS were found to have a 2-fold increased risk of metabolic syndrome in a cross-sectional study from Turkey that reported the prevalence of PCOS under NIH and Androgen Excess Society criteria as 6.1\% and 15.3\%, respectively (9).

\section{Onset of Clinical Manifestation in PCOS}

Although clinical manifestations of PCOS usually occur during puberty with the onset of maturation of the hypothalamicpituitary-ovarian axis, the female foetus might be programmed to develop PCOS in adult life due to exposure to an excess of androgen in foetal life because of the genetic or environmental factors or a combination of them $(10,11)$.

Genetically-determined hypersecretion of the androgens from the ovary is proposed to be the primary event that leads to the development of PCOS by favouring excess luteinising hormone (LH) secretion and insulin resistance (12). However, insulin resistance with or without a genetic mutation may be the initiator, followed by hyperandrogenism, as is seen in hyperandrogenism-insulin resistance-acanthosis nigricans (HAIR-AN) syndrome $(13,14)$. Insulin resistance is an important contributing factor to abnormal steroidogenesis in PCOS. Insulin acts with $\mathrm{LH}$ to increase androgen production and promotes $\mathrm{LH}$ binding to the receptors. Hyperinsulinaemia stimulates insulin like growth factor-I (IGF-I) pathway in theca cells of the ovary by cross-reacting with the receptors for IGF. This state blocks the down-regulation of androgens by LH surge and leads to the hyperandrogenic environment in the ovary. Treatments include weight loss, which lowers insulin levels, restores ovulation and lowers the ovarian androgen levels (15).

\section{Genetic Factors Related to PCOS}

No consistent abnormality was detected in the chromosomal studies of the patients with PCOS but genetic studies of the families of the affected women showed a high incidence of affected relatives, indicating both $\mathrm{X}$-linked and autosomal dominant inheritance (16). Moreover, there are data implying the linkage of adolescent PCOS with paternal metabolic syndrome (17). In order to identify the genetic factors resulting in the hereditary nature of PCOS, genes encoding enzymes involved in androgen synthesis (serine hyperphosphorylation of P450c17, 21hydroxylase (CYP21) mutation), or protein transducers of insulin signals (excessive phosphorylation of IR-beta, insulin receptor substrate (IRS) proteins, IRS-1 (Gly(972)Arg) and IRS-2 (Gly(1057) Asp) have been analysed $(18,19)$. However, the role of genetic polymorphisms or mutations in the pathogenesis of PCOS is still under investigation and further research is required.

\section{Metabolic Manifestations of PCOS}

In the past, PCOS was considered only a hyperandrogenic disorder which can lead to infertility. Current data show that the diagnosis of PCOS is related to an increased risk of metabolic disturbances like insulin resistance (IR), hyperinsulinism (HI), impaired glucose intolerance, increased risk of type 2 diabetes in later life due to $\mathrm{HI}$ and IR, obesity, subclinical atherosclerosis, vascular dysfunction, metabolic syndrome and cardiovascular disease. Because central obesity is associated with hyperandrogenism and cardiovascular diseases, the Endocrine Society also recommends screening adolescents with PCOS for central obesity by body mass index (BMI) and waist circumference measurements (4).

For this reason, primary care for metabolic and cardiovascular risks and defining preventive methods in women with PCOS are the definite goals of the treatment of PCOS (20).

The American Heart Association categorised the cardiovascular risks of women with PCOS. Patients with obesity, especially 
abdominal adiposity, hypertension, dyslipidaemia, subclinical vasculopathy, impaired glucose tolerance, a family history of familial cardiovascular disease $(<55$ years of age in a male relative and $<65$ years of age in a female relative) and cigarette smoking were categorised as at risk for PCOS-related cardiovascular disease. Those who had metabolic syndrome, type 2 diabetes, and overt renal and vascular disease were categorised as the high-risk population according to American Heart Association criteria (21).

\section{Metabolic Markers of Metabolic Syndrome in PCOS}

In a recent study, the relationship of activins and follistatins with metabolic markers in PCOS was investigated (22). Serum follistatin levels were found to be elevated in PCOS patients, independent of age and BMI. Activin levels were found to be similar in both groups with and without PCOS. In this study, follistatin was related to markers of adiposity and lipids in both women with and without PCOS. Activins A and B have various reproductive and metabolic actions in females. They stimulate follicular growth and also inhibit androgen production in the theca cells of the ovary (23). On the other hand, follistatin neutralises activin activity, and inhibits follicle stimulating hormone (FSH) secretion and folliculogenesis. Follistatin is also a promoter in the inflammation process, which has been shown to initiate insulin resistance and diabetes (24). For this reason, the increased levels of follistatin might inhibit folliculogenesis by increasing ovarian androgen production. In order to understand the possible role of follistatin in the pathogenesis of PCOS, further research is necessary.

Women with PCOS usually have abdominal adiposity and an increased risk of metabolic syndrome. Central obesity leads to the secretion of various adipocyte-derived peptide hormones, named adipocytokines. Adiponectin is the major adipocytokine that is secreted from the visceral fat cells. The presence of adiponectin impairs glucose tolerance and is an important predictor of metabolic syndrome (25). Ghrelin is also another peptide hormone which has a role in energy metabolism and low levels were reported to be associated with insulin resistance and diabetes (26). Adiponectin and ghrelin levels were negatively correlated with androgen levels in some trials, thus supporting the relationship between the hyperandrogenaemic state of PCOS and metabolic risk factors (27). In this study, obese hirsute women with PCOS were found to have lower adiponectin levels than obese controls and women with PCOS also had lower ghrelin levels than weight-matched controls. Panidis et al. (28) reported an inverse correlation between ghrelin levels and androgen levels. They found ghrelin levels to be lower in women with PCOS and also lower still in patients with hyperandrogenaemia.

Ersan et al. (29) investigated the relationship between adipocytokines and metabolic syndrome in pre-menopausal women with PCOS; adiponectin was found to be significantly lower and leptin was significantly higher in patients with PCOS and metabolic syndrome.

However, in a recent study, the levels of metabolic risk markers like adiponectin, leptin and ghrelin were measured in obese adolescents with and without PCOS; all were found to be similar in both groups (30). The investigators concluded that the presence of PCOS alone does not result in a higher risk of metabolic syndrome in adolescents. As expected, adiponectin levels were negatively correlated and leptin positively correlated with BMI.

\section{Prevalence of Metabolic Syndrome in Adolescents with PCOS}

After the first description of metabolic syndrome by Reaven in 1988, many organisations like the National Cholesterol Education Program (NCEP), the International Disease Federation (IDF), the World Health Organisation (WHO), and the European Group for the Study of Insulin Resistance published the diagnostic criteria for syndrome (31). Among these, the 2001 Third Report of NCEP's Adult Treatment Panel is the most widely accepted definition as all risk factors can be easily and routinely measured using diagnostic criteria. However, the WHO and European Group for the Study of Insulin Resistance definition includes routinely unmeasured criteria like insulin resistance and microalbuminaemia. According to the NCEP criteria, the presence of any three criteria defines metabolic syndrome. Those criteria are denoted as follows: Central obesity (waist circumference $\geq 88 \mathrm{~cm}$ in women), serum triglycerides $\geq 150 \mathrm{mg} / \mathrm{dL}$, serum HDL concentration $<50 \mathrm{mg} / \mathrm{dL}$ in women, systemic hypertension $\geq 130 / 85 \mathrm{mmHg}$, and fasting plasma glucose level $\geq 100 \mathrm{mg} / \mathrm{dL}$ (32).

In Turkey, Çalışkan et al. (33) investigated the frequency of metabolic syndrome according to different diagnostic criteria in women with PCOS. All criteria used identified higher metabolic syndrome in patients with PCOS than in controls. However, the highest prevalence (26\%) was demonstrated by using IDF criteria due to the lower cut-off values for waist circumference and fasting glucose levels in these definitions.

In a U.S. population survey, the overall presence of metabolic syndrome was identified as $22 \%$ and gradually increased with age. The prevalence of metabolic syndrome in the US population was determined as $6.7 \%$ in the twenties, increasing to $43.5 \%$ in the sixties (34). Aging increases the visceral fat deposition, which worsens the glucose and lipid metabolism. Contrary to US data, the prevalence of metabolic syndrome in Italy was determined as $2.4 \%$ in general population in the $20 \mathrm{~s}$ and this was found to increase to nearly $5 \%$ in the 40 s. This is probably due to the lower incidence of obesity in Italy. However, the prevalence was 3-fold higher in young women with PCOS (35). There is an ongoing debate regarding the definition of metabolic syndrome in adolescents in the literature. Hormonal fluctuations which lead to metabolic changes during transition to adolescence may mimic the features of metabolic syndrome. In one study of 1098 adolescents, nearly half of the adolescents initially diagnosed as metabolic syndrome lost this diagnosis during the three years observation period (36). The reason for this change might be due to the lack of objective and consistent criteria for identifying metabolic syndrome in adolescents. In 2007, Jolliffe et al. (37) reported the age-specific metabolic syndrome criteria for adolescents by linking them to the NCEP and 
ITP adult criteria with growth chart modelling. Similar to the growth charts used to monitor height and weight in children, the growth curve method is used to easily identify metabolic syndrome. Growth curves for waist circumference, blood pressure, serum high-density lipoprotein (HDL) and triglyceride (TG) concentrations were defined, but no growth curve was set for fasting glucose level due to the constant level $(100 \mathrm{mg} / \mathrm{dL})$ from 12 to 20 years of age in this study.

The International Diabetes Federation put forward a new definition to identify children and adolescents with metabolic syndrome in 2007 . The definition is easily applicable in clinical practice and categorised according to the age group (32). The IDF suggests that the diagnosis of metabolic syndrome should not be used for children younger than 10 years of age. If the waist circumference is $\geq 90$ percentile in the children younger than 10 years of age, weight reduction is recommended. For children aged between 10 and 16, the presence of abdominal obesity according to waist circumference percentiles with any two clinical features (elevated TG, low levels of HDL, elevated blood pressure, elevated fasting glucose) is sufficient for the diagnosis of metabolic syndrome. For children aged 16 years and older, it is recommended to use the adult criteria.

In a study conducted on Iranian adolescents with PCOS, the presence of metabolic syndrome was nearly three-fold higher than the control group (33.3\% and $11.2 \%$, respectively) (38). The frequency of metabolic syndrome components like hypercholesterolemia, hypertriglyceridemia and elevated blood pressure was significantly higher in obese adolescents with PCOS. However, there was no significant difference among metabolic parameters between the non-obese adolescents with or without PCOS.

The National Heart, Lung, and Blood Institute Growth and Health Study (NGHS) identified predisposing factors for developing metabolic syndrome in childhood. After 10 years followup, waist circumference and serum triglycerides were identified as predictive factors of metabolic syndrome in girls aged 9 and 10 years. For every $1 \mathrm{~cm}$ increase in waist circumference at year 2 , the metabolic syndrome risk increased by $7.4 \%$ and for every increase of $1 \mathrm{mg} / \mathrm{dl}$ in triglycerides level, the metabolic syndrome risk increased by 1.3\% (39). In one cohort study with a 25 year follow-up, it was found that self-reported cardiovascular disease was observed more often (19.4\%) in adults who had clinical features of metabolic syndrome as children than in those who did not (1.5\%) (40).

\section{Pathogenesis of Metabolic Syndrome in Adolescent PCOS Women}

Obesity is an important predictor of metabolic syndrome. Even normal weight females with PCOS are found to have a 50\% greater body fat level than normal weight females (41). In a study of 205 adolescents ( $\leq 20$ years), the presence of metabolic risk factors and metabolic syndrome was investigated. The prevalence of being overweight or obese was significantly higher in adolescents with PCOS compared with those without (60\% vs. 18\%) (42).

The relationship between features of PCOS and features of metabolic syndrome were investigated in a cohort study of ado- lescent Australian girls; neither menstrual disturbances nor PCO morphology were found to be related to insulin resistance. BMI is the most prominent factor for the presence of IR. When it is estimated independent of obesity, an elevated free testosterone level is the most prominent indicator for the presence of the insulin resistance. Nearly one third of the adolescents with PCOS (35.3\%) were found to be at risk of metabolic syndrome, whereas only $15.5 \%$ of adolescents without PCOS had this risk (43).

In a study of 469 South Asian women with PCOS, the relationship between different phenotypes of PCOS and metabolic syndrome was investigated. Although normal cycling hyperandrogenic women (Hyperandrogenism and PCO morphology) had a significantly lower incidence of obesity than the other phenotypes, the prevalence of metabolic syndrome was similar in all phenotypes. In contrast to a large USA study, the data of which reported a two-fold increased risk of metabolic syndrome in hyperandrogenic women, this study did not reveal any link between plasma testosterone and the occurrence of metabolic syndrome (44).

In a recent study, we analysed the endocrine and cardiovascular risk profile differences between 139 women with main PCOS phenotypes in Turkey. Among 4 PCOS phenotypes, patients with hyperandrogenism and PCOS (HA\&PCOS) had the lowest carotid intima thickness; low-density lipoprotein cholesterol (LDL-C), total cholesterol and BMI were also found to be lower in this group. For this reason, this phenotype is said to have the lowest cardiovascular risk compared to other phenotypes (2).

Amato et al. (45) conducted a study in order to verify a method for distinguishing the metabolic health of women of reproductive age with PCOS. The visceral adiposity index is suggested as an easy and successful method for the assessment of metabolically unhealthy women and the detection of cardiometabolic risk factors. The visceral adiposity index (VAI) is calculated by a formula using waist circumference (WC), BMI, TG and HDL levels.

BMI is the most important variables which precludes the presence of metabolic syndrome. In a cross-sectional study of overweight and obese adolescents, the presence of PCOS did not add any weight to the presence of the features of metabolic syndrome. In this study, 53\% of PCOS and 55\% of the control group obese adolescents met the diagnostic criteria of metabolic syndrome (46).

\section{Management of PCOS in Adolescents}

In an internet survey that questioned specialists about their clinical approaches to PCOS in North America, the percentage of the respondent's patients with PCOS who were $<18$ years was found to be $53 \%$ and the percentage of respondent's PCOS patients who were obese was found to be $65 \%$ (7). The percentage of specific tests used for the initial diagnosis was highly variable; nearly $80 \%$ of the specialists evaluated thyroid stimulating hormone (TSH) and prolactin (PRL), whereas 17\% of them evaluated sex hormone binding globulin (SHBG). For the evaluation of metabolic syndrome features, nearly $60 \%$ of specialists searched lipid profile and fasting glucose level, $41 \%$ 
investigated fasting insulin levels and $25 \%$ assessed haemoglobin A1C. In a large cohort of normal weight women with PCOS in Austria, the efficiency of diabetes screening tests like HbAlc and fasting glucose level were evaluated. The true incidence of diabetes was $12.8 \%$, which was determined by using oral glucose tolerance testing (OGTT); in contrast, when using only $\mathrm{HbA} 1 \mathrm{C}$ and fasting glucose levels, only $3.2 \%$ and $5.2 \%$ of patients, respectively, had a diagnosis of pre-diabetes. In conclusion, the authors stated that although the OGTT is time consuming, neither fasting glucose nor HbAlc can be used as an alternative screening test for pre-diabetes in PCOS (47).

Weight loss with a low-fat and low-carbohydrate diet has improved menstrual function in obese adolescents with PCOS in a recent study (48). Marsh et al. (49) compared the low glycaemic index diet and conventional healthy diet in patients with polycystic ovary syndrome; after similar weight losses, women who consumed the low glycaemic diet showed more regular menstrual cycles. In a cohort study, the presence of insulin resistance in lean women with PCOS is investigated; none of the lean PCOS women were found to have insulin resistance and, as a conclusion, the routine performance of OGTT in lean women with PCOS is not recommended (50). The most recent guideline of the Endocrine Society also recommends exercise therapy in overweight and obese adolescents in order to reduce the cardiovascular risk. However, in normal weight women with PCOS, exercise therapy alone is not sufficient for treatment (4).

In our recent study, obesity was found to be negatively correlated to health-related quality of life in patients with PCOS. We analysed the health quality profiles of infertile women according to different PCOS phenotypes in this study (51). PCOS phenotype 1 patients (Hyperandrogenism and anovulation) had significantly higher BMI and hirsutism scores in comparison to other phenotypes and also showed significantly lower healthrelated quality of life scores.

Studies have demonstrated higher rates of many psychiatric disorders like anxiety, depression and eating disorders in adults and adolescents with PCOS $(4,52)$. The impact of hirsutism on the development of psychiatric problems are debatable (53). Given the high prevalence of anxiety and depression in women with PCOS, adolescents with PCOS should also be screened for these in their history and should be referred to psychiatrists for treatment.

Sleep disturbances like obstructive sleep apnoea appear to be a common complaint in women with PCOS. Sleep disordered breathing and daytime sleepiness were also more common in women with PCOS than in controls (54). In particular, the presence of hyperandrogenism and obesity are the suspected contributing factors for sleep disorders. Given the high prevalence of obstructive sleep apnoea in women with PCOS, especially overweight and obese adolescents should be evaluated for possible obstructive sleep apnoea symptoms and referred for polysomnography when suspected (4).

\section{Management of Metabolic Syndrome in Adolescents}

The prevention of adiposity, especially abdominal adiposity, is the primary target for the prevention of metabolic syndrome.
For weight loss, lifestyle modifications like a restricted calorie diet and exercise are recommended as the first-line treatment by the recently published Endocrine Society guideline. There is no evidence that supports the superiority of one type of diet (4). The Mediterranean low fat diet that is high in fruit and vegetables has been found to improve glucose intolerance, insulin resistance, vascular endothelial function and lipid metabolism (55). The current recommended exercise program is a daily minimum of 30 minutes of moderate-intensity physical activity. Weight loss of about 5 to $10 \%$ of baseline weight is recommended in order to correct the abnormal glucose metabolism, but a cut-off level of fasting glucose has not yet been determined in patients with metabolic syndrome without diabetes.

Currently, studies in the literature investigating metabolic syndrome in children and adolescents with PCOS are quite limited; for this reason, it is difficult to define the long-term risk of cardiovascular disease and diabetes. Two randomised controlled studies demonstrated that the use of metformin improves the ovulation, hyperandrogenisim and abnormal lipid profile in adolescents with PCOS. Based on these data, the Endocrine Society recommended the use of metformin for the treatment of metabolic syndrome (4). However, early intervention with lifestyle changes such as increased physical activity, diet and smoking cessation may ameliorate these features in especially overweight and obese adolescents with PCOS. Metformin may be a choice of treatment when lifestyle modification is ineffective in obese adolescents.

\section{Conclusion}

The diagnosis of PCOS in adolescents is not easy due to a variety of diagnostic criteria and overlapping symptoms of pubertal development. However, the early diagnosis of PCOS is also important for identifying potential metabolic and cardiovascular risks during this period. Sleep disorders and psychiatric disease may also be diagnosed and treated earlier during the adolescence period. We know that especially obese adolescents with PCOS have an increased risk of metabolic syndrome. Although there is debate on the diagnostic criteria of metabolic syndrome during adolescence, abdominal obesity is the most prominent predictor of metabolic syndrome during childhood and adolescence. Therefore, early interventions with a Mediterranean diet, increased physical activity and smoking cessation are recommended in order to prevent metabolic syndrome, especially in obese adolescents with PCOS.

\section{Ethics Committee Approval: N/A. \\ Informed Consent: $N / A$.}

Peer-review: Externally peer-reviewed.

Author contributions: Concept - D.A.C., B.D.; Design - D.A.C., B.D.; Supervision - B.D.; Resource - B.D., D.A.C.; Materials - B.D., D.A.C.; Data Collection\&/or Processing - D.A.C.; Analysis\&/or Interpretation - D.A.C., B.D.; Literature Search - D.A.C.; Writing D.A.C., B.D.; Critical Reviews - B.D.

Conflict of Interest: No conflict of interest was declared by the authors. 
Financial Disclosure: The authors declared that this study has received no financial support.

\section{References}

1. Stein IF. Amenorrhea associated with bilateral polycystic ovaries. Am J Obstet Gynecol 1935; 29: 181.

2. Dilbaz B, Ozkaya E, Cinar M, Cakir E, Dilbaz S. Cardiovascular disease risk characteristics of the main polycystic ovary syndrome phenotypes. Endocrine 2011; 39: 272-7. [CrossRef]

3. Azziz R, Carmina E, Dewailly D, Diamanti-Kandarakis E, EscobarMorreale HF, Futterweit W, et al. The Androgen Excess and PCOS Society criteria for the polycystic ovary syndrome: the complete task force report. Fertil Steril 2009; 91: 456-88. [CrossRef]

4. Legro RS, Arslanian SA, Ehrmann DA, Hoeger KM, Murad MH, Pasquali R, Welt CK. Diagnosis and treatment of polycystic ovary syndrome: an endocrine society clinical practice guideline. J Clin Endocrinol Metab 2013; 98: 4565-92. [CrossRef]

5. Carmina E, Oberfield SE, Lobo RA. The diagnosis of polycystic ovary syndrome in adolescents. Am J Obstet Gynecol 2010; 203: 201.e1-5.

6. Norman RJ, Dewailly D, Legro RS, Hickey TE. Polycystic ovary syndrome. Lancet 2007; 370: 685-97. [CrossRef]

7. Bonny AE, Appelbaum H, Connor EL, Cromer B, DiVasta A, GomezLobo V, et al. Clinical variability in approaches to polycystic ovary syndrome. J Pediatr Adolesc Gynecol 2012; 25: 259-61. [CrossRef]

8. Hardy TSE, Norman RJ. Diagnosis of adolescent polycystic ovary syndrome. Steroids. 2013; 78: 751-4. [CrossRef]

9. Yildiz BO, Bozdag G, Yapici Z, Esinler I, Yarali H. Prevalence, phenotype and cardiometabolic risk of polycystic ovary syndrome under different diagnostic criteria. Hum Reprod 2012; 27: 3067-73. [CrossRef]

10. Xita N, Tsatsoulis A. Review: fetal programming of polycystic ovary syndrome by androgen excess: evidence from experimental, clinical, and genetic association studies. J Clin Endocrinol Metab 2006; 91: 1660-6. [CrossRef]

11. Franks S, McCarthy MI, Hardy K. Development of polycystic ovary syndrome: involvement of genetic and environmental factors. Int $\mathrm{J}$ Androl 2006; 29: 278-85. [CrossRef]

12. Abbott DH, Dumesic DA, Franks S. Developmental origin of polycystic ovary syndrome - a hypothesis. J Endocrinol 2002; 174: 1-5. [CrossRef]

13. Barbieri RL, Hornstein MD. Hyperinsulinemia and ovarian hyperandrogenism. Cause and effect. Endocrinol Metab Clin North Am 1988; 17: 685-703.

14. Omar HA, Logsdon S, Richards J. Clinical profiles, occurrence, and management of adolescent patients with HAIR-AN syndrome. Scientific World Journal 2004; 4: 507-11. [CrossRef]

15. Kaltsas GA, Mukherjee JJ, Jenkins PJ, Satta MA, Islam N, Monson $\mathrm{JP}$, et al. Menstrual irregularity in women with acromegaly. J Clin Endocrinol Metab 1999; 84: 2731-5. [CrossRef]

16. Battaglia C, Regnani G, Mancini F, Iughetti L, Flamigni C, Venturoli S. Polycystic ovaries in childhood: a common finding in daughters of PCOS patients. A pilot study. Hum Reprod 2002; 17: 771-6. [CrossRef]

17. Leibel NI, Baumann EE, Kocherginsky M, Rosenfield RL. Relationship of adolescent polycystic ovary syndrome to parental metabolic syndrome. J Clin Endocrinol Metab 2006; 91: 1275-83. [CrossRef]

18. Pugeat M. Genetics of the polycystic ovarian syndrome and therapeutic perspectives. Rev Med Chir Soc Med Nat Iasi 2000; 104: 11-9.
19. Ehrmann DA, Tang X, Yoshiuchi I, Cox NJ, Bell GI. Relationship of insulin receptor substrate-1 and -2 genotypes to phenotypic features of polycystic ovary syndrome. J Clin Endocrinol Metab 2002; 87: 4297-300. [CrossRef]

20. Wild RA, Carmina E, Diamanti-Kandarakis E, Dokras A, EscobarMorreale HF, Futterweit W, et al. Assessment of cardiovascular risk and prevention of cardiovascular disease in women with the polycystic ovary syndrome: a consensus statement by the Androgen Excess and Polycystic Ovary Syndrome (AE-PCOS) Society. J Clin Endocrinol Metab 2010; 95: 2038-49. [CrossRef]

21. Mosca L. Guidelines for prevention of cardiovascular disease in women: a summary of recommendations. Prev Cardiol 2007; 10: 19-25. [CrossRef]

22. Teede H, Ng S, Hedger M, Moran L. Follistatin and activins in polycystic ovary syndrome: relationship to metabolic and hormonal markers. Metabolism 2013; 62: 1394- 400. [CrossRef]

23. Knight PG, Satchell L, Glister C. Intra-ovarian roles of activins and inhibins. Mol Cell Endocrinol 2012; 359: 53-65. [CrossRef]

24. Sjöholm A, Nyström T. Inflammation and the etiology of type 2 diabetes. Diabetes Metab Res Rev 2006; 22: 4-10. [CrossRef]

25. Fasshauer M, Paschke R. Regulation of adipocytokines and insulin resistance. Diabetologia. 2003; 46: 1594-603. [CrossRef]

26. Pöykkö SM, Kellokoski E, Hörkkö S, Kauma H, Kesäniemi YA, Ukkola O. Low plasma ghrelin is associated with insulin resistance, hypertension, and the prevalence of type 2 diabetes. Diabetes 2003; 52: 2546-53. [CrossRef]

27. Glintborg D, Andersen M, Hagen C, Frystyk J, Hulstrøm V, Flyvbjerg A, Hermann AP. Evaluation of metabolic risk markers in polycystic ovary syndrome (PCOS). Adiponectin, ghrelin, leptin and body composition in hirsute PCOS patients and controls. Eur J Endocrinol 2006; 155: 337-45. [CrossRef]

28. Panidis D, Farmakiotis D, Koliakos G, Rousso D, Kourtis A, Katsikis I, et al. Comparative study of plasma ghrelin levels in women with polycystic ovary syndrome, in hyperandrogenic women and in normal controls. Hum Reprod 2005; 20: 2127-32. [CrossRef]

29. Ersan F, Arslan E, Çorbacıoğlu Esmer A, Aydın S, Gedikbaşı A, Gedikbaşı A, Alkış I, Ark C. Prediction of metabolic syndrome in women with polycystic ovary syndrome. J Turk Ger Gynecol Assoc 2012; 13: 178-183. [CrossRef]

30. Kale-Gurbuz T, Akhan SE, Bastu E, Telci A, lyibozkurt AC, Topuz S. Adiponectin, leptin and ghrelin levels in obese adolescent girls with polycystic ovary syndrome. J Pediatr Adolesc Gynecol 2013; 26: 27-30. [CrossRef]

31. Reaven GM. Banting lecture 1988. Role of insulin resistance in human disease. Diabetes 1988; 37: 1595-1607. [CrossRef]

32. Zimmet P, Alberti KG, Kaufman F, Tajima N, Silink M, Arslanian S, et al. The metabolic syndrome in children and adolescents - an IDF consensus report. Pediatr Diabetes 2007; 8: 299-306. [CrossRef]

33. Çalışkan E, Kılıç T, Bodur H, Zeteroğlu Ş. The frequency of metabolic syndrome in women with polycystic ovaries at reproductive ages and comparison of different metabolic syndrome diagnostic criteria. J Turk Ger Gynecol Assoc 2007; 8: 402-7.

34. Ford ES, Giles WH, Dietz WH. Prevalence of the metabolic syndrome among US adults: findings from the third National Health and Nutrition Examination Survey. JAMA 2002; 287: 356-9. [CrossRef]

35. Carmina E, Napoli N, Longo RA, Rini GB, Lobo RA. Metabolic syndrome in polycystic ovary syndrome (PCOS): lower prevalence in southern Italy than in the USA and the influence of criteria for the diagnosis of PCOS. Eur J Endocrinol 2006; 154: 141-5. [CrossRef]

36. Goodman E, Daniels SR, Meigs JB, Dolan LM. Instability in the diag- 
nosis of metabolic syndrome in adolescents. Circulation 2007; 115: 2316-22. [CrossRef]

37. Jolliffe CJ, Janssen I. Development of age-specific adolescent metabolic syndrome criteria that are linked to the Adult Treatment Panel III and International Diabetes Federation criteria. J Am Coll Cardiol 2007; 49: 891-8. [CrossRef]

38. Rahmanpour H, Jamal L, Mousavinasab SN, Esmailzadeh A, Azarkhish K. Association between polycystic ovarian syndrome, overweight, and metabolic syndrome in adolescents. J Pediatr Adolesc Gynecol 2012; 25: 208-12. [CrossRef]

39. Morrison JA, Friedman LA, Harlan WR, Harlan LC, Barton BA, Schreiber GB, Klein DJ. Development of the metabolic syndrome in black and white adolescent girls: a longitudinal assessment. Pediatrics 2005; 116: 1178-82. [CrossRef]

40. Morrison JA, Friedman LA, Gray-McGuire C. Metabolic syndrome in childhood predicts adult cardiovascular disease 25 years later: the Princeton Lipid Research Clinics Follow-up Study. Pediatrics 2007; 120: 340-5. [CrossRef]

41. Kirchengast $\mathrm{S}$, Huber J. Body composition characteristics and body fat distribution in lean women with polycystic ovary syndrome. Hum Reprod 2001; 16: 1255-60. [CrossRef]

42. Roe AH, Prochaska E, Smith M, Sammel M, Dokras A. Using the androgen excess-PCOS society criteria to diagnose polycystic ovary syndrome and the risk of metabolic syndrome in adolescents. J Pediatr 2013; 162: 937-41. [CrossRef]

43. Hart R, Doherty DA, Mori T, Huang RC, Norman RJ, Franks S, et al. Extent of metabolic risk in adolescent girls with features of polycystic ovary syndrome. Fertil Steril 2011; 95: 2347-53. [CrossRef]

44. Wijeyaratne CN, Seneviratne Rde A, Dahanayake S, Kumarapeli V, Palipane E, Kuruppu N, et al. Phenotype and metabolic profile of South Asian women with polycystic ovary syndrome (PCOS): results of a large database from a specialist Endocrine Clinic. Hum Reprod 2011; 26: 202-13. [CrossRef]

45. Amato MC, Guarnotta V, Forti D, Donatelli M, Dolcimascolo S, Giordano C. Metabolically healthy polycystic ovary syndrome (MH-PCOS) and metabolically unhealthy polycystic ovary syndrome (MU-PCOS): a comparative analysis of four simple methods useful for metabolic assessment. Human Reprod 2013; 28: 1919-28. [CrossRef]

46. Rossi B, Sukalich S, Droz J, Griffin A, Cook S, Blumkin A, et al. Prevalence of metabolic syndrome and related characteristics in obese adolescents with and without polycystic ovary syndrome. J Clin Endocrinol Metab 2008; 93: 4780-6. [CrossRef]
47. Lerchbaum E, Schwetz V, Giuliani A, Obermayer-Pietsch B. Assessment of glucose metabolism in polycystic ovary syndrome: HbAlc or fasting glucose compared with the oral glucose tolerance test as a screening method. Hum Reprod 2013; 28: 2537-44. [CrossRef]

48. Ornstein RM, Copperman NM, Jacobson MS. Effect of weight loss on menstrual function in adolescents with polycystic ovary syndrome. J Pediatr Adolesc Gynecol 2011; 24: 161-5. [CrossRef]

49. Marsh KA, Steinbeck KS, Atkinson FS, Petocz P, Brand-Miller JC. Effect of a low glycemic index compared with a conventional healthy diet on polycystic ovary syndrome. Am J Clin Nutr 2010; 92: 83-92. [CrossRef]

50. Stovall DW, Bailey AP, Pastore LM. Assessment of insulin resistance and impaired glucose tolerance in lean women with polycystic ovary syndrome. J Womens Health (Larchmt) 2011; 20: 37-43. [CrossRef]

51. Dilbaz B, Çınar M, Özkaya E, Vanlı Tonyalı N, Dilbaz S. Health Related Quality of Life Among Different PCOS Phenotypes of Infertile Women. J Turk Ger Gynecol Assoc 2012; 13: 247-52. [CrossRef]

52. Özsoy S. Psychiatric status and approach in polycystic ovary syndrome and hirsutism. Turkiye Klinikleri J Endocrin-Special Topics 2009; 2: 126-30.

53. Tunç S, Tanrıkulu L, Özcan L, Yenicesu O, Akın Su F, Subası B. Psychopathological symptoms in adolescents with hirsutismus. The Journal of Gynecology - Obstetrics and Neonatology 2013; 10: 1518-21.

54. Vgontzas AN, Legro RS, Bixler EO, Grayev A, Kales A, Chrousos GP. Polycystic ovary syndrome is associated with obstructive sleep apnea and daytime sleepiness: role of insulin resistance. J Clin Endocrinol Metab 2001; 86: 517-20. [CrossRef]

55. Esposito K, Marfella R, Ciotola M, Di Palo C, Giugliano F, Giugliano $\mathrm{G}$, et al. Effect of a mediterranean-style diet on endothelial dysfunction and markers of vascular inflammation in the metabolic syndrome: a randomized trial. JAMA 2004; 292: 1440-6. [CrossRef]

56. Zawadzki J, Dunaif A. Diagnostic Criteria for Polycystic Ovary Syndrome: towards a Rational Approach. In: Dunaif A, Givens J, Haseltine F, Merriam G, editors. Polycystic Ovary Syndrome. Blackwell Scientific Publications, Cambridge. 1992.p.377.

57. Rotterdam ESHRE/ASRM-Sponsored PCOS Consensus Workshop Group. Revised 2003 consensus on diagnostic criteria and longterm health risks related to polycystic ovary syndrome. Fertil Steril 2004; 81: 19-25. [CrossRef] 